\title{
Experimental advances in superlubricity
}

\author{
Quanshui ZHENG ${ }^{1,2, *}$, Ze LIU ${ }^{1}$ \\ ${ }^{I}$ Department of Engineering Mechanics, CNMM, APL, and ${ }^{2}$ National Key Lab of Tribology, Tsinghua University, Beijing 100084, China \\ Received: 17 May 2014 / Accepted: 10 June 2014 \\ (C) The author(s) 2014. This article is published with open access at Springerlink.com
}

\begin{abstract}
Superlubricity, or structural lubricity, is a state that has two contacting surfaces exhibiting no resistance to sliding. This effect has been theoretically described to be possible between two completely clean single crystalline solid surfaces. However, experimental observations of superlubricity were limited to nanoscale and under high vacuum or inert gas environments even after twenty years since the concept of superlubricity has been suggested in 1990. In the last two years, remarkable advances have been achieved in experimental observations of superlubricity ranging from micro-scale to centimeters and in ambient environment. This study aims to report a comprehensive understanding of the superlubricity phenomenon.
\end{abstract}

Keywords: Superlubicity; microscale; macroscale; ambient condition; mechanism

\section{Introduction}

Superlubricity may occur when two single crystalline surfaces slide over each other in a dry incommensurate contact. This effect was first proposed by Hirano and coauthors in 1990 [1,2]. For example, graphite has a laminar structure consisting of hexagonal graphene bound by van der Waals interaction. The contact between two graphene surfaces is considered to be in commensurate registry, when their lattice orientations are the same as shown in Fig. 1(a). Because of the hexagonal structure, the commensurate registry repeats every 60 degrees in relative rotation. At any lattice misfit orientation, however, the contact is considered to be in an incommensurate registry, as shown in Fig. 1(b). The shear strength of graphite in commensurate registry is reported to be about $140 \mathrm{MPa}$ [3], while that in an incommensurate registry is theoretically predicted to possibly vanish [1]. Similar properties hold for other laminar hexagonal structures, when the interlayer binding forces is via van der Waals interactions and other contributions to friction like electrons can be neglected. Consequently, the essential feature of superlubricity state is that apparently

* Corresponding author: Quanshui ZHENG.

E-mail: zhengqs@tsinghua.edu.cn the friction suddenly and dramatically drops to zero or near zero when the contact shifts from a commensurate to an incommensurate registry. We call this characteristic the superlubricity essential feature (SEF), as schematically illustrated in Fig. 1 (c).

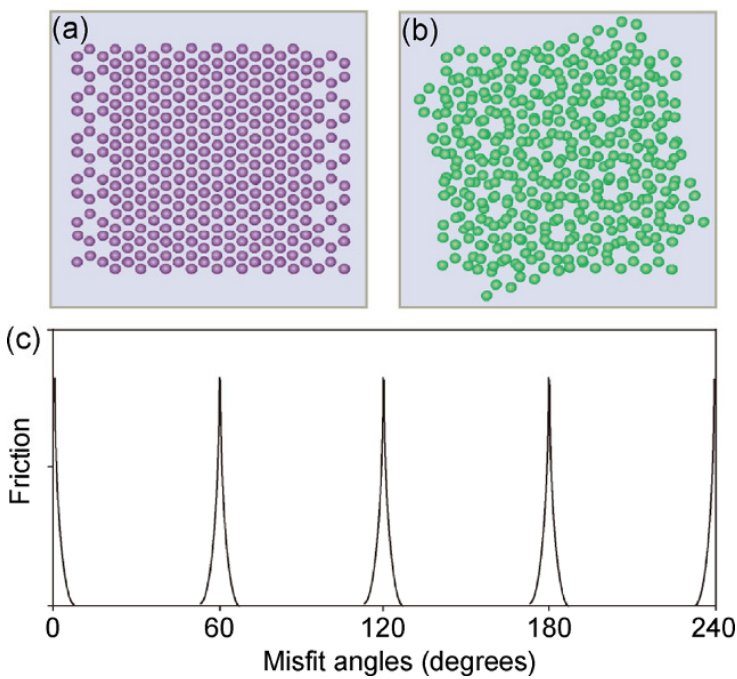

Fig. 1 Two graphene layers contact in commensurate registry (a) and in incommensurate registry (b). (c) Superlubricity essential feature (SEF) between graphene layers, where the high friction will drop quickly to zero or near zero when their contact is shifted from a commensurate to an incommensurate registry. For graphenes, the commensurate registry repeats every 60 degrees in relative rotation. 
The first experimental attempt to observe superlubricity was made in 1991 by Hirano and coauthors [2] using a crystalline muscovite mica, which is a laminar hexagonal material. The observed friction was found to indeed depend on the lattice misfit angles (Fig. 2), but not the SEF that is necessary for superlubricity. Besides, the measured minimum friction force of $2.2 \times 10^{-4} \mathrm{~N}$ under a normal force $1.2 \times 10^{-3} \mathrm{~N}$ results in a friction coefficient of 0.18 . This is too high to be regarded as superlubricity. Previously experiments on friction between two crystalline surfaces are justified on the basis of friction anisotropy $[4,5]$ and not superlubricity.

Hirano et al. attempted to demonstrate another experimental observation of superlubricity by measuring the friction between a tungsten tip with $\mathrm{W}(011)$ surface and $\mathrm{Si}(001)$ surface under ultrahigh vacuum conditions using scanning tunneling microscopy (STM) [6]. The contact area was estimated to be of the order of few $\mathrm{nm}^{2}$. According to their study, no friction signal for the tungsten tip could be detected for incommensurate contact with the STM resolution of about $3 \times 10^{-9} \mathrm{~N}$. Given the fact that the frictional force of $3 \times 10^{-9} \mathrm{~N}$ for a nanometer-sized contact corresponds to shear strength of the order of $1 \mathrm{GPa}$, saying that this

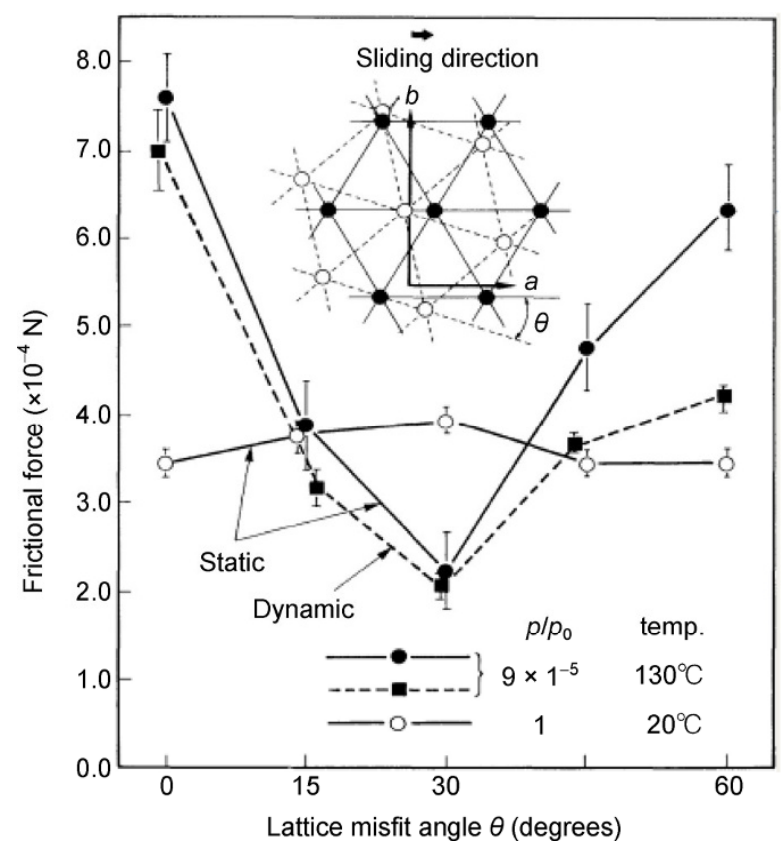

Fig. 2 Measured friction force between two mica surfaces as function of lattice misfit angle between them (Reproduced with permission from Ref. [2]. Copyright American Physical Society, 1991). experiment confirms the existence of superlubricity is not convincing.

Molybdenum disulphide $\left(\mathrm{MoS}_{2}\right)$ is also a well-known lamellar solid lubricant with a hexagonal structure. When testing a sputtered $\mathrm{MoS}_{2}$ coating exempt of impurities such as carbon, oxygen, and water vapor, Martin et al. [7] found that the friction coefficient could be down to the $10^{-3}$ range, which is at least one order of magnitude lower than the values reported so far. The observed ultralow friction was attributed to the change of an inter-crystallite slip system by an effect of friction induced lattice orientation, in which the basal planes are aligned in the sliding direction and disordered around the $c$-axis. This reorientation could happen because of the observed nanosize of the grains. Consequently, the $\mathrm{MoS}_{2}$ surfaces in friction contact would be of dimensional nanosized polycrystalline in nature. As a result, the majority of the contact region would be incommensurate, leading to superlubricity. However, a minor portion of the contact area, although ultralow, can possibly be in commensurate registry, contributing the majority of the observed friction. In other words, the observed ultralow friction is not a direct evidence of superlubric phenomenon.

In view of the above mentioned situation and the argument that electronic contributions may also play a role in determining the friction [8,9], Müser [10] suggested to use the terminology of superlubricity as kinetic friction coefficient in the order of magnitude $10^{-3}$ or lesser. The superlubricity induced by incommensurate registry was then renamed as "structural lubricity".

A clear evidence of superlubricity was provided by Dienwiebel and co-workers [11] using a frictional force microscope with a resolution of lateral forces down to $15 \mathrm{pN}$, which allowed quantitative tracking of the forces on the scanning tip in three directions. By attaching a graphite nanoflake to a nanotip, they measured the friction force between the graphite layers as function of misfit angles. As shown in Fig. 3, there are two pulses around the orientations of $0^{\circ}$ and $60^{\circ}$ that correspond to two commensurate registries, while the friction is negligible at the misfit angles. These observations agreed well with the superlubricity essential feature. Since the diameter of the graphite 

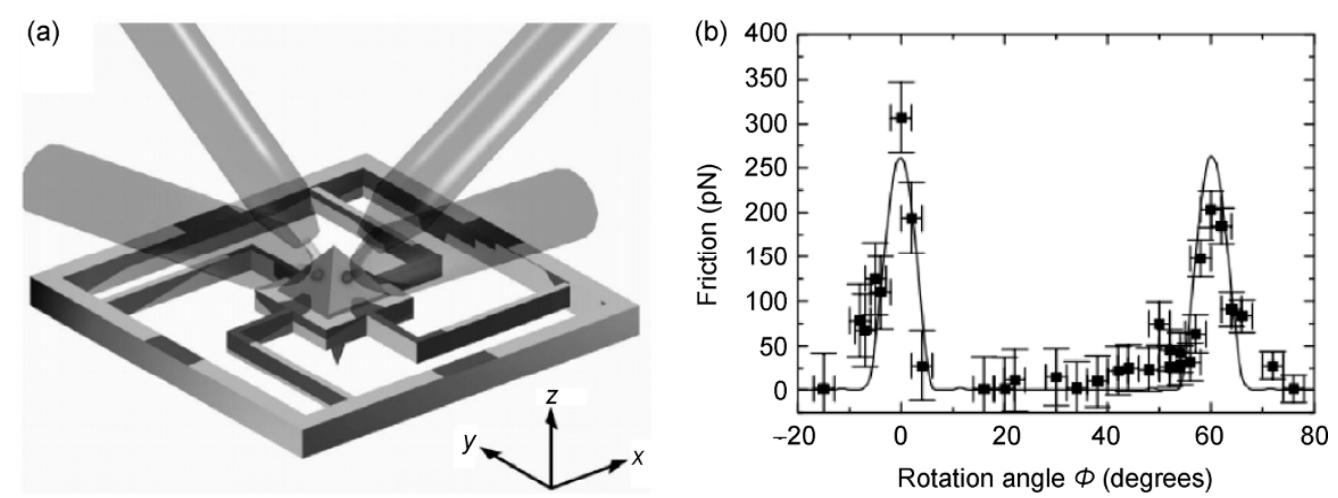

Fig. 3 (a) A home-built FFM, which can track forces on the scanning tip in three directions. (b) Measured average friction force versus rotation angle between graphite nanoflakes and graphite substrate. Two narrow peaks of high friction were observed at $0^{\circ}$ and $61^{\circ}$ respectively (Reproduced with permission from Ref. [11]. Copyright American Physical Society, 2004).

nanoflake was estimated to be about $1.4 \mathrm{~nm}$ [12], the measured minimum friction force of $15.2 \pm 15 \mathrm{pN}$ corresponds to a shear strength of $9.9 \pm 9.7 \mathrm{MPa}$, which is two orders in magnitude lower than the reported values [6]. Furthermore, considering the average value $(\sim 20 \mathrm{nN})$ of normal force in their experiments, the calculated friction coefficient is as small as $0.00076 \pm$ 0.00075 .

Recently, some studies have reported superlubricity related phenomena, like graphene nanoflakes sliding on graphene [13], scaling law of superlubricity [14], and negative friction behavior $[15,16]$.

Overall, the observations of superlubricity, even after twenty years of its first inception in 1990, were limited to nanoscale ranged contact areas, and under high vacuum conditions or inert gas environments. According to a theoretical study [11], for sufficiently large contacts, superlubricity might break down if the two lattices are not perfectly rigid. Besides, a network of misfit dislocations might form between them, which will result in friction. As commented by Urbakh et al. $[17,18]$, there exists a large gap between the current understanding of superlubricity on the microscopic and macroscopic scales. To the best of our knowledge, all observations of structural superlubricity until 2011 were limited to nanoscale regime and under high vacuum or inert gas conditions, as briefly reviewed in the previous section. This greatly hinders the utilization of superlubricity phenomenon for practical applications.

In the following Section 2, we review the recent advances that clearly demonstrate the observation of superlubricity from micrometer to centimeter scales under ambient conditions. These observations [3, 19-24] are essentially based on a novel technology established by us with the aid of a so-called self-retracting motion phenomenon. A deeper understanding on the underlying mechanism, as compared with that reported in the previous studies, on the observation of superlubricity at larger scale is also presented in Section 2. Following that, in the Section 3, we elucidate two important applications of the observed superlubricity phenomenon. Finally, we provide brief summary and future perspectives in Section 4.

\section{Observations of superlubricity in larger scale under ambient conditions}

\subsection{Challenges}

In order to realize superlubricity in larger scale, having two large atomically smooth surfaces is necessary. However, this is not the sole criterion to observe superlubricity. The biggest challenge to realize superlubricity is perhaps establishing contact between two solid surfaces that are completely clean [10, 25, 26], given the fact that the absorption and/or contaminants can easily hinder superlubricity [25]. To the best of our knowledge, in all the experiments reported before 2012 for observing superlubricity, the two surfaces in contact were placed together. Under this condition, avoiding absorption is hard, particularly 
in case of larger samples such as that considered by Hirano et al. [2] between two crystalline muscovite mica flakes.

\subsection{Self-retraction phenomenon in graphite}

The first observation of superlubricity in micrometer scale and under ambient conditions was realized in graphite [22], based on a novel property of selfretracting motion discovered by Zheng et al. [19]. The self-retraction experiments were performed on square graphite mesas covered with a $\mathrm{SiO}_{2}$ film of thickness about $100 \mathrm{~nm}$, as illustrated in Fig. 4(a). The height of the mesas was about $200 \mathrm{~nm}$, with side lengths (sizes), $L$, varying in the range of 0.5 to $5 \mu \mathrm{m}$. The technique adopted for preparing these mesas has been explained in detail elsewhere [19]. The experiment on each tested mesa was performed by controlling the probe of a micromanipulator to laterally push the upper edge or horizontally rub on the top surface of the $\mathrm{SiO}_{2}$ cover. Using this method, we could successfully slip out flakes from the tested mesas to various prescribed suspended positions. Each flake examined consisted of the $\mathrm{SiO}_{2}$ cover and an attached graphite flake slipped out from the mesa, as illustrated in Fig. 4(b) and as verified from SEM (bottom image of Fig. 4(b)). We found that some of the suspended graphite/ $\mathrm{SiO}_{2}$ flakes under test can automatically and fully retract back onto the top of their respective base mesas immediately upon releasing the applied forces by removing the probe away from the flakes. The observation that the retracting motion still occurred even though the probe was removed away in the direction opposing the retraction motion direction (see Figs. 4(c)-4(f) and the EPAPS movie [19]) excluded the qualm that the retracting motion could be caused by the adhesion or electrostatic force between the flakes and the removing probe.

Interestingly, we found that the self-retracting motion not only occurred under high vacuum condition, but also under ambient conditions or even under water. Furthermore, we found that the self-retraction probability, namely the ratio of self-retractable flakes to the non-self-retractable ones increases with decrease in the size of the flakes or with increase in the etching of the mesas' height. More quantitatively, the selfretraction probability is $100 \%$ for 1 and $2 \mu \mathrm{m}$ mesas, while it is $87 \%, 33 \%$, and $13 \%$ for $3.0,3.5$, and $5.0 \mu \mathrm{m}$ mesas for one batch of samples, respectively.

\subsection{Superlubricity in micro-scale graphite}

As mentioned before, the greatest challenge in realizing superlubricity at larger scale and under ambient condition is the difficulties associated in obtaining two atomically flat surfaces at larger scale that can contact with each other without absorptions and/or contaminants between them. According to the study reported by Liu et al. [20], the self-retraction property in graphite provides a novel solution to this challenges.

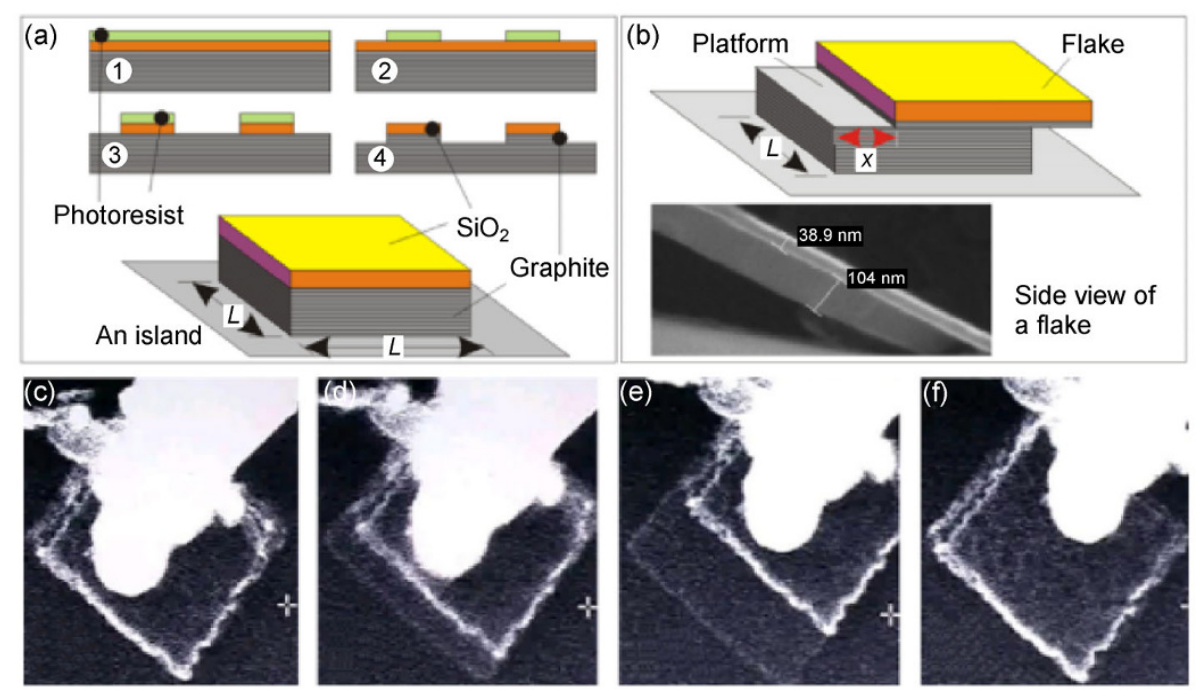

Fig. 4 Fabricating graphite mesas capped with $\mathrm{SiO}_{2}$ film (a) and the observation of self-retracting motion of flipped out graphite/SiO flakes (b) when released by lift-up probe (c)-(f) [19]. 
As illustrated in Fig. 4 (b), shearing out of a flake for a distance $x$ from a graphite mesa of size $L$ will generate two new graphite surfaces with the total area of $2 L x$. In the meantime, it will increase the total free energy $U=2 L x \gamma$, where $\gamma$ denotes the surface energy of the graphite. The driven force is therefore equal to $F=-\mathrm{d} U / \mathrm{d} x=-2 \gamma \mathrm{L}$. We observed self-retracting motion in mesas with $L$ ranging from $0.5 \mu \mathrm{m}$ to $10 \mu \mathrm{m}$ and for $x$ ranging from almost zero to near $L$. Thus, the maximum shear strength against the self-retracting motion is bounded by $\tau^{\text {upper }}<2 \gamma / L \approx 0.04 \mathrm{MPa}$, where the value of $\gamma$ is known to be about $0.1-0.2 \mathrm{~J} / \mathrm{m}^{2}$ $[21,27,28]$.

We found that there were certain orientations at which the self-retracting motion was locked. These locked orientations have clear hexagonal symmetry. The self-retracting motion could occur at almost any orientation, except in those locked orientations, as shown in Fig. 5. The nearest self-retractable orientation to a locked one was observed to be $1^{\circ}$. The shear resistive shear force at any self-retractable orientation was measured to be three orders of magnitude lower than those at the locked orientation. The latter agrees well with the basal plane shear strength of single crystalline graphite [3]. Given the hexagonal nature of the graphite structure, these properties signify a clear picture that the locked orientations correspond to commensurate registries, while the self-retractable orientations correspond to incommensurate registries. The sudden and dramatic (three orders) drop of the resistance to sliding indicates that the self-retracting motion is an effect of superlubricity.

\subsection{Mechanism of the size effects of the self- retraction motion}

The sliding surfaces of all the fully self-retractable samples were found to be atomically smooth without even a single atomic height step, as observed by using STM (Fig. 6 (a)). In contrast, all the non-self-retractable sheared surfaces had various defects (Fig. 6(b)).

To gain deeper insights on the underlying mechanism, we performed electron backscattered diffraction (EBSD) analysis of a fresh surface made of the same type of highly oriented pyrolytic graphite (HOPG) that was used to create the mesas [22]. The corresponding results are shown in Fig. 6(c), wherein the domains with different colors correspond to different grains. The crystal orientation map (IPF map) at the same region shows that the different grains have the same crystal orientation along [0001] (out-of-plane) [22], indicating the high degree of orientation in HOPG. The grain sizes range from a few micrometers to about $60 \mu \mathrm{m}$, which is about three orders of magnitude larger than the grain thicknesses that typically range from few to dozens of nanometers [29]. In other words, the HOPGs used in the experiments are polycrystals with paperlike or very thin grains (Inset shown in Fig. 6(c)).

Taking into consideration the polycrystalline nature of HOPG, a proposed simple stone wall (or layer of rock) model for the graphite mesas [22] was illustratively shown in Fig. 7. Herein, each stone corresponds to a single-crystalline graphite grain. That is, the graphene layers in each stone are contact in commensurate registry, while the interfaces between the grains perpendicular to the $c$-axis (out-of-plane) are in

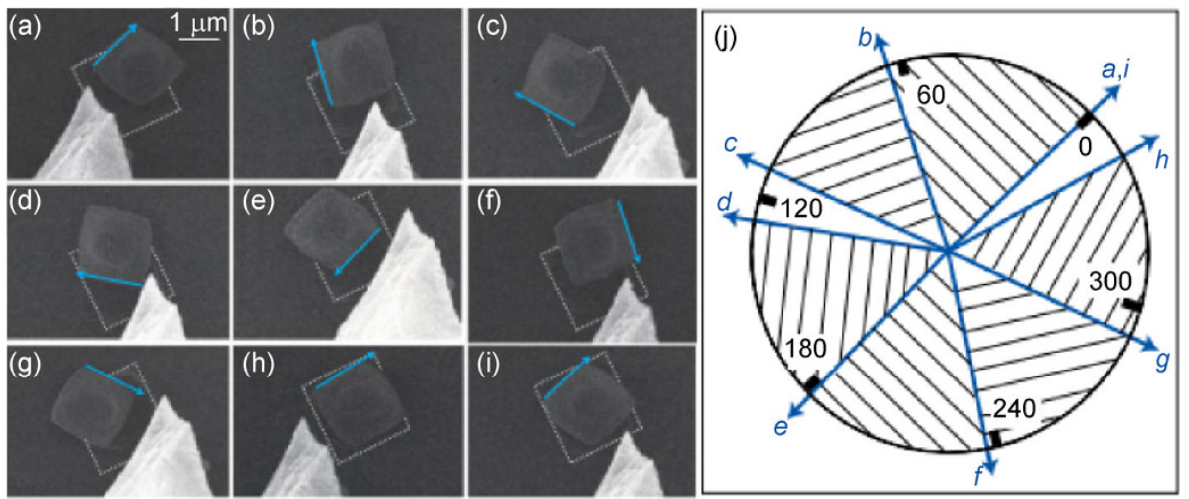

Fig. 5 Orientation dependent self-retraction motion [22]. The "lock-in" directions in (a)-(i) clearly indicating the $60^{\circ}$ symmetry (j). 

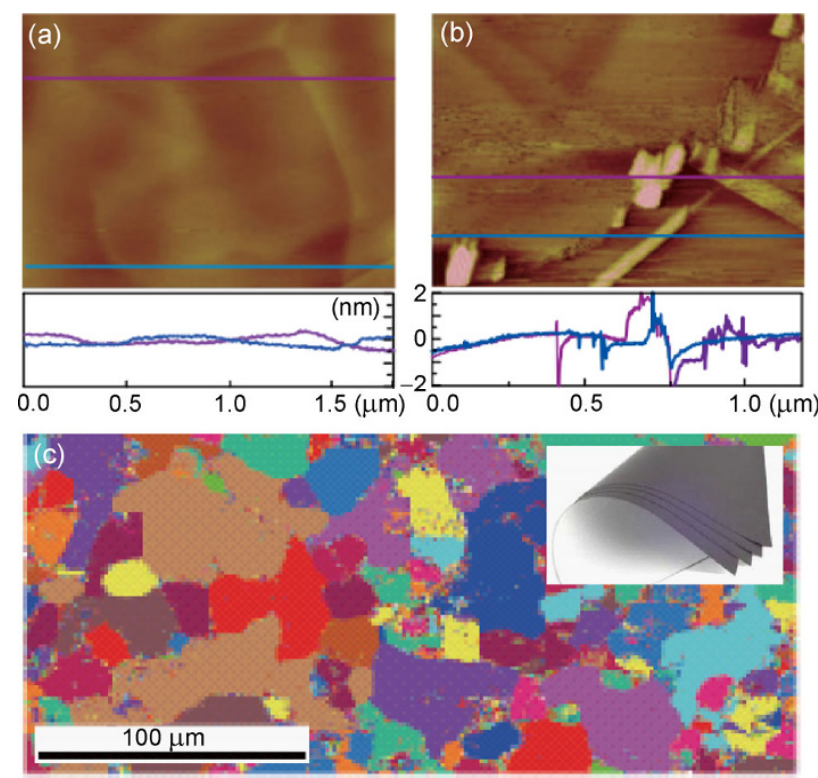

Fig. 6 Atomic smooth sliding surface for self-retractable samples (a) and various surface defects in non-self-retractable samples (b) revealed by STM. EBSD mapping a fresh surface of HOPG (ZYH grade) showed the grain size distribution (c) and the corresponding IPF map in this region also indicated a paper-like grain (inset of (c)) structures [22].

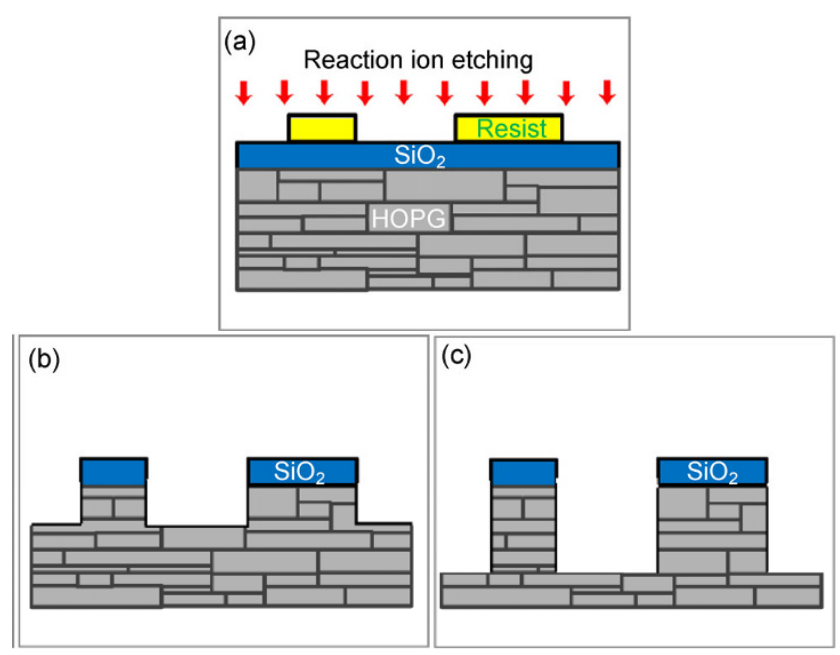

Fig. 7 Illustration of the proposed stone wall polycrystalline structure for fabricated graphite $/ \mathrm{SiO}_{2}$ mesas [22]. (a) Illustrative of fabricating graphite/ $\mathrm{SiO}_{2}$ mesas. (b) The probability of existence of a single grain boundary across the whole mesa is higher for smaller mesa than that for larger mesa. (c) Increasing the etching of mesa height gives a higher probability of the existence of a single grain boundary across the whole mesa even though for larger mesa.

incommensurate registry. If the graphite mesas are small of the order of $1 \mu \mathrm{m}$, as the smallest size used in our experiments, then the cracked interfaces following a totally incommensurate interface is highly possible.
Accordingly, the sheared graphite flake can undergo complete self-retraction motion, which is exactly as observed in our experiments [19,22]. On the other hand, for larger graphite mesas of the order of $20 \mu \mathrm{m}$ used in our experiments, it is virtually impossible for the cracked interface to be in a totally incommensurate contact. As a result, steps (Fig. 7(b)) or partially commensurate contact areas will appear in the sliding surfaces that might hinder the self-retracting motion. However, for a higher mesa, there is a high probability of having a single grain boundary across the whole mesa, as schematically illustrated in Fig. 7(c). Indeed, the probability of size-dependent self-retracting motion was observed to increase for higher mesas in our experiments [22].

\subsection{Persistence of superlubricity in graphite at high speed}

For the first time, we measured the speed of selfretracting motion (SRM) in graphite by using a home-made optical in-situ recording system with knife-edge technology [30, 23], as shown in Figs. 8(a) and $8(\mathrm{~b})$. For samples of size $L=3 \mu \mathrm{m}$, the maximum SRM speed was recorded to be about $25 \mathrm{~m} / \mathrm{s}$ (Fig. 8(c)) at the temperature of $235^{\circ} \mathrm{C}$. This suggests that the superlubricity can persist in graphite, even at such a high speed. The SRM speed was found to depend remarkably on temperature from the $\mathrm{mm} / \mathrm{s}$ range at room temperature to $25 \mathrm{~m} / \mathrm{s}$ at $235^{\circ} \mathrm{C}$ (Fig. $8(\mathrm{~d})$ ). These observations suggest that defects, such as edge defects, can affect superlubricity, while thermal activation can weaken this effect [23].

\subsection{Macroscopic superlubricity in multi-walled carbon nanotubes}

The first observation of macroscopic superlubricity was realized during the technique adopted to produce centimeter-long double-/triple-walled carbon nanotubes (DWCNTs/TWCNTs) with perfect atomic structures and while visualizing the dynamic behavior of CNTs by depositing $\mathrm{TiO}_{2}$ nanoparticles onto the surface of CNTs [31]. Zhang et al. [24] showed that the inner shell of a carbon nanotube can self-retract back to its outer shell (Fig. 9). The inter-shell friction of the DWCNTs was measured by using a silicon nanorod 

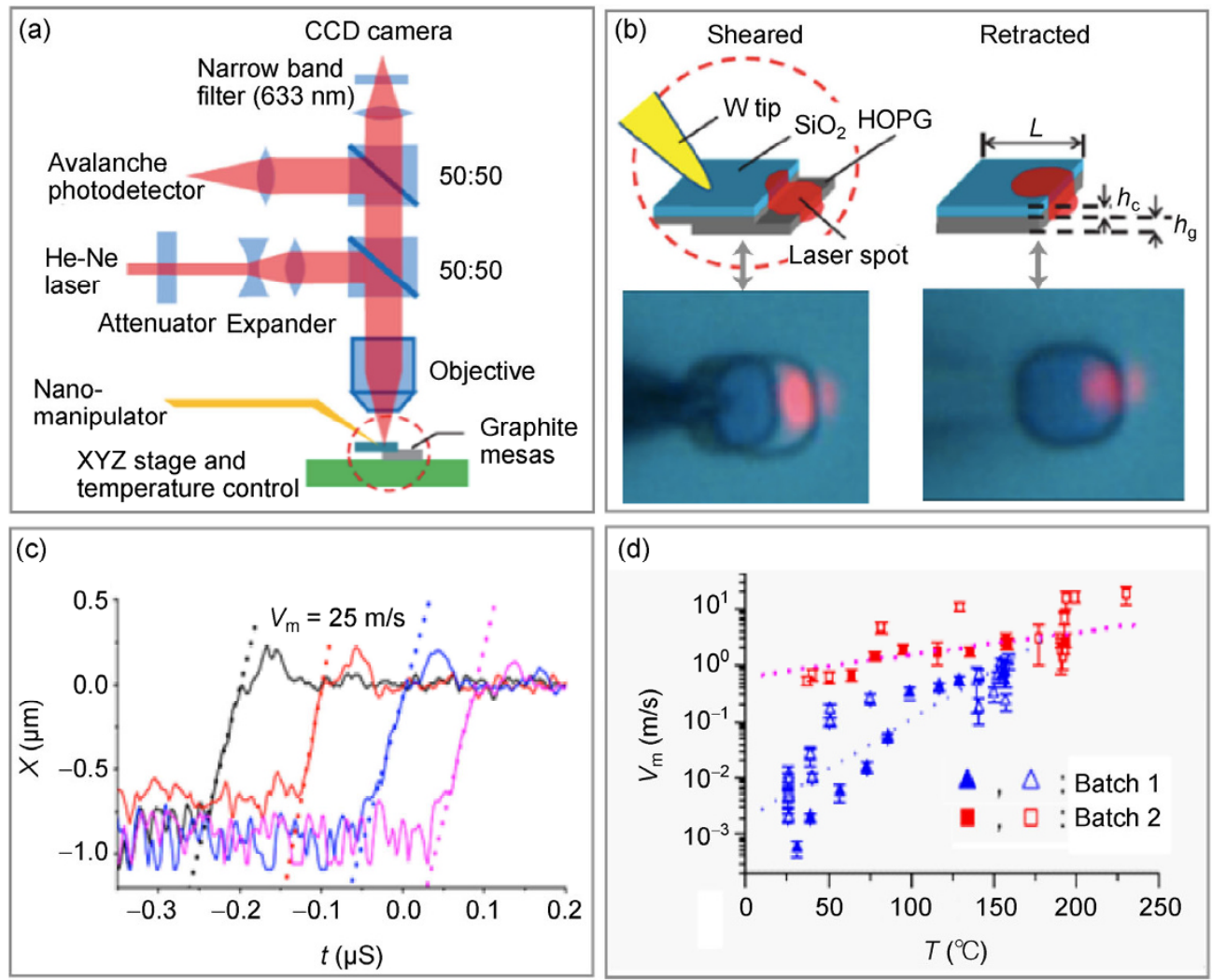

Fig. 8 The self-retracting dynamics (displacements versus time) was recorded based on a home-built optical in-situ recording system by applying knife-edge technology [23]. The measured maximum of self-retracting speed can as high as $25 \mathrm{~m} / \mathrm{s}$; several SRMs for this mesa are shown, to demonstrate the reproducibility of the process. The speeds obtained from fitting the linearly rising section of the curve are $21 \mathrm{~m} / \mathrm{s}, 25 \mathrm{~m} / \mathrm{s}, 17 \mathrm{~m} / \mathrm{s}$, and $20 \mathrm{~m} / \mathrm{s}$ from left to right (c). While for some samples, the measured speed is lowered by four orders in magnitude but it will increase when heating the sample (d).



Fig. 9 Illustration of self-retraction motion of an ultra-long DWCNTs and corresponding optical images showing inner-shell sliding and self-retracting along its out shell [24]. 
as a force cantilever (Figs. 10(a) and 10(b)) [24]. The calculated shear strength between carbon nanotube shells is as small as 2.6 Pa, implying that the sliding friction is almost vanished while the contact area is up to $77 \mu \mathrm{m}^{2}$. Based on this measured value of interlayer shear strength and assuming that it is almost of the same order for interlayer shear strength of graphene layers with incommensurate contact, the theoretical maximum size of graphite mesa that can keep selfretracting motion is determined to be as large as $0.15 \mathrm{~m}$.

The main mechanism underlying the origin of superlubricity between an extruded core tube and its outer holder is that the end of the outer tube can clean the inner core when the core tube retracts back.

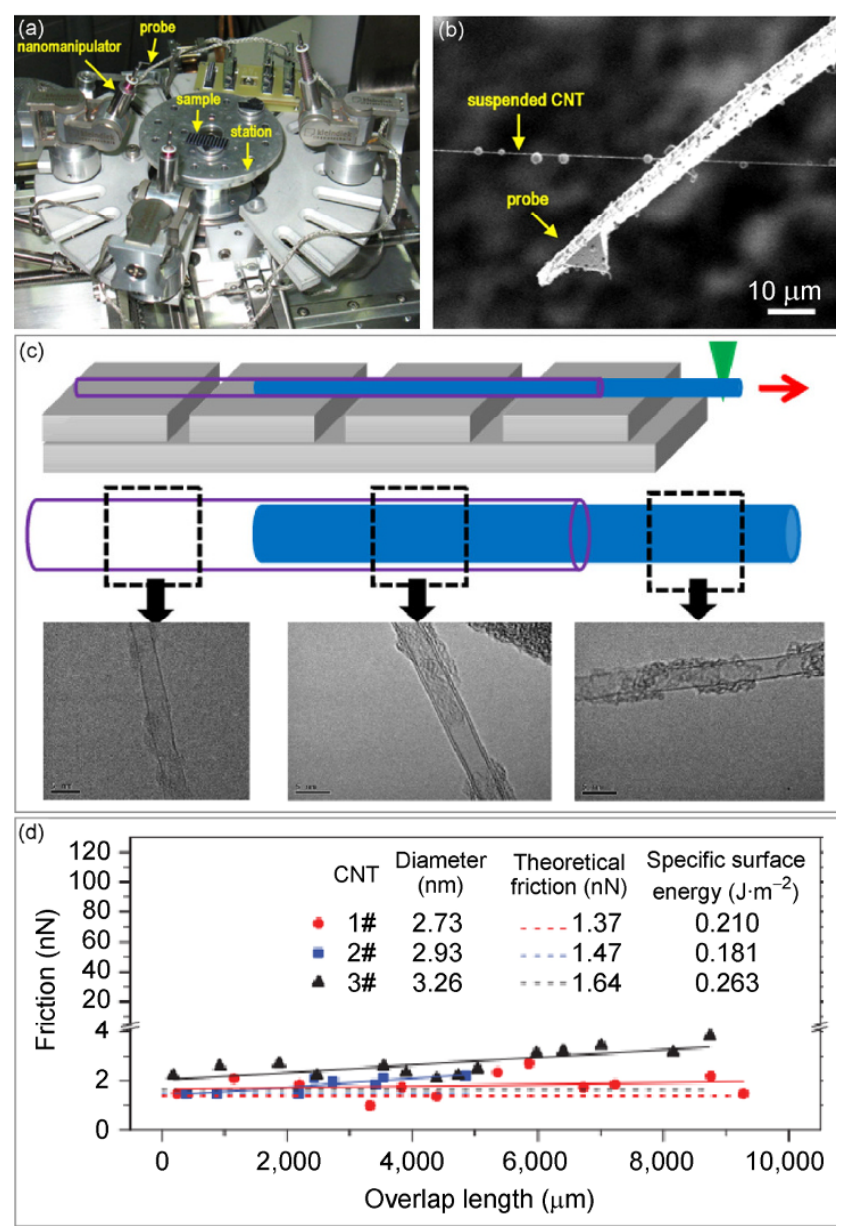

Fig. 10 By using a nanomanipulator equipped SEM and a silicon nanorod-based force sensor, the intershell friction of DWCNTs was measured as low as $\sim 2 \mathrm{nN}$ and almost independent on the overlap length [24].

\section{Two representative applications of super- lubricity}

\subsection{Nanoeraser}

Intriguingly, the self-retractable graphite mesas demonstrated in this study exhibited high repeatability [22] under ambient environments or even under water. Superlubricity persisting in such heavy contaminating environment is strange.

Liu et al. found that the contaminants such as carbon deposits and adhesive residue on the graphite basal planes can be readily cleaned by sliding a graphite flake on the graphite plane (Fig. 11) [20]. The mechanism underlying this effect can be explained as follows. When compared with the null resistance of a graphite microflake sliding on a completely clean graphite basal plane (with incommensurate contact), the intra-layer strength and rigidity of graphene are extremely high. Therefore, the first few graphene layers on the contact surface of the graphite flake act as a shovel, thereby cleaning the contaminants in front of the sliding direction of the flake. The van der Waals adhesion between the flake and the graphite mesa, and the very weak bending rigidity of the graphene layer will facilitate the first few graphene layers to closely contact the graphite mesa, preventing the contaminants from entering into the interspace between the two contacted graphene layers. A detailed explanation of the so-called nanoeraser effect is provided in Ref. [20].
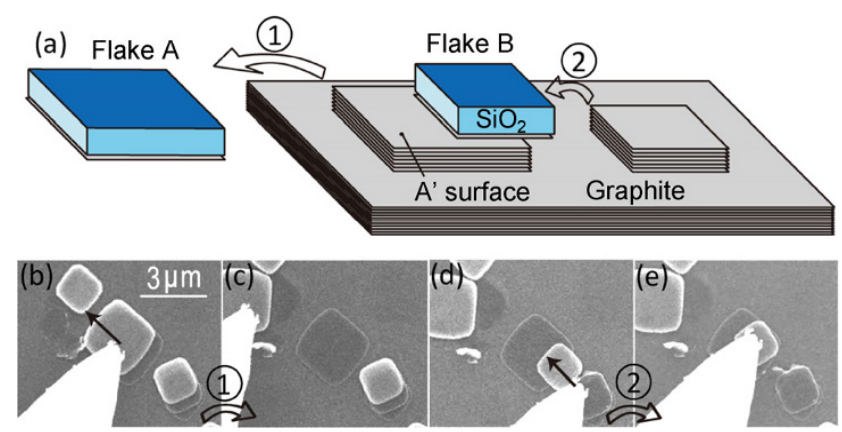

Fig. 11 In situ cleaning amorphous carbon layers away from graphite surface in SEM: After pushing graphite/ $\mathrm{SiO}_{2}$ flake A away, the totally exposed A' surface will be covered with a thin amorphous carbon layers which is induced by electron beam irradiation, while when we transferred another graphite/ $\mathrm{SiO}_{2}$ flake $\mathrm{B}$ into the exposed A' surface and sliding it there, the flake B can recover self-retraction motion in the A' surface.[20] 


\subsection{Basal plane surface energy and interlayer cohesive energy of graphite}

As mentioned earlier, the driving force for the selfretracting motion of a sheared flake is $2 \gamma L$, where $\gamma$ is the free surface energy of the basal plane of the graphite. Or equivalently, $2 \gamma$ can be approximated as the interlayer cohesive energy between the two basal planes (graphene layers). As one of the most fundamental material properties of graphite, however, cohesive energy had not been directly measured until the recent works of Liu et al. [21].

Due to the stone wall structure of HOPG, as discussed in Section 2.4, upon shearing a graphite mesa of typical size $L=3 \mu \mathrm{m}$ and height $h=200 \mathrm{~nm}$ made from a HOPG, there may exist several crossthe-mesa planes that are incommensurate contact interfaces between two single-crystalline graphite grains. This structural feature enables to shear out several graphite flakes that would be self-retractable. As illustrated in Fig. 12, after shearing off a selfretractable flake (Fig. 12(a)), rotating the flake until it gets locked at a commensurate registry (Fig. 12(b)) is possible. The shear force required to generate this cracked plane mainly consists of the self-retraction force $(2 \gamma L)$, resistance to break the edge linkages that may be formed during the mesa fabrication, and static friction. Since this force is much lower than that required for cracking a commensurate contact, shearing this cracked mesa again can shear off another self-retractable flake (Fig. 12(c)). Again, this newly cracked plane can be locked by rotating it into a commensurate contact, which forms a stable, non-selfretractable, and multi-cracked structure as shown in the representative SEM image (Fig. 12 (e)). Because of the very thin nature of the flakes, the suspended flakes can be easily bent and adhered to the base cracked plane (Fig. 12(d)). The range and shape of the bent region are the results of a competition between the cohesive energy and the bending energy. Thus, with the measured information on the range and shape of the bent region, the value of cohesive energy was estimated to be $0.19( \pm 0.01) \mathrm{J} / \mathrm{m}^{2}$. This can serve as a benchmark for other theoretical and experimental works related to graphite/graphene systems.
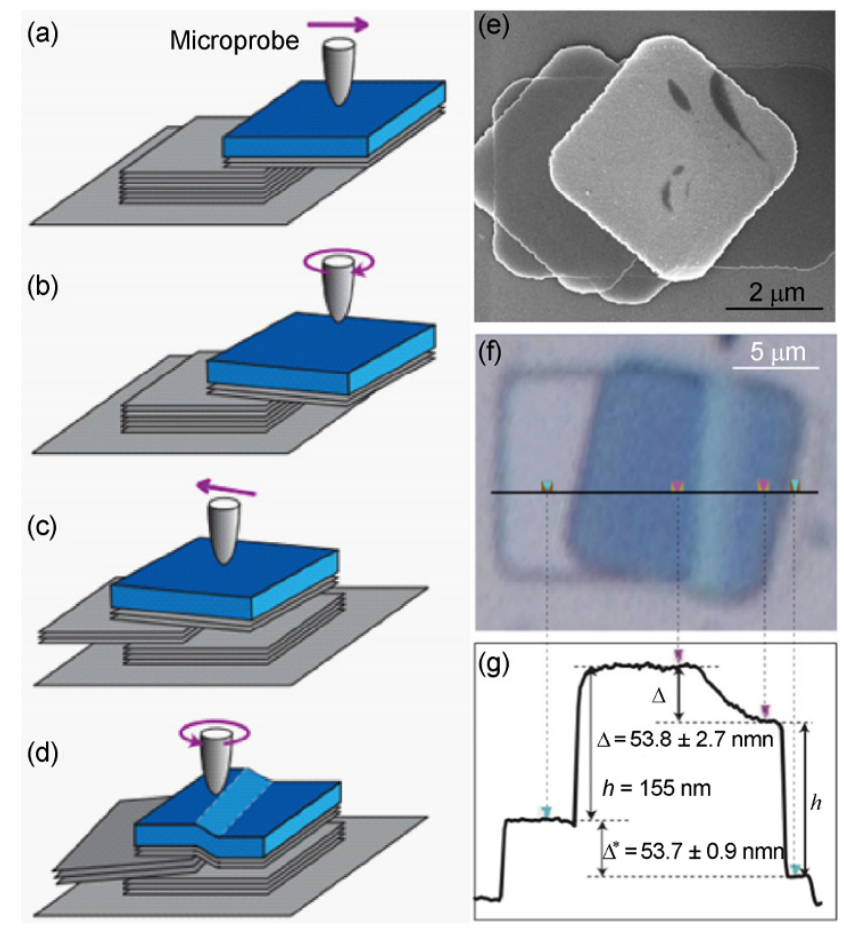

Fig. 12 Illustrate processes of in situ fabricating and assembling a graphite $/ \mathrm{SiO}_{2}$ flake spanned across a graphite nanostep (a)-(d) based on the self-retracting mechanism and one fabricated example of playing-cards like graphite nanoplates by this method. After that, AFM is employed to quantify the geometries which would be used for adhesion energy calculation. [21]

\section{Concluding remarks and perspectives}

We developed a novel technology based on the phenomenon of self-retracting motion to observe superlubricity at microscale in graphite (HOPG) and at macroscale in CNTs (double- or triple-walled). Superlubricity at this larger scale was found to persist under ambient conditions, in water (for HOPG), and at high speeds (up to $25 \mathrm{~m} / \mathrm{s}$ for HOPG).

On the basis of the novel property of self-retracting motion, we also developed a technology for obtaining absolutely clean surfaces.

Further studies are underway on the experimental observations of superlubricity in several laminatestructured materials, such as $\mathrm{MoS}_{2}$, Mica, and recently created single-crystalline $2 \mathrm{D}$ materials.

We speculate that the larger scale single-crystalline 2D materials will play a key role in future applications of superlubricity [32], such as in new types of hard disks $[10,33]$ and in oscillators based on van der Waals 
interaction [34, 35]. Other applications of structural superlubricity based on graphite and graphene include inertia device [36], memory device [37] and shuttle [38].

On a final note, the limited resolution of the instruments restricts the applicability of superlubricity in experimental measurements. Consequently, there still lacks valid definition of superlubricity for experimentally identifying superlubricity and comparing superlubricity between different experiments.

\section{Acknowledgements}

The financial supports from the National Natural Science Foundation of China (NSFC) (Grant No. 10832005) and the the National Key Basic Research (973) Program of China (Grants Nos. 2007 CB936803 and 2013CB934200) are acknowledged.

Open Access: This article is distributed under the terms of the Creative Commons Attribution License which permits any use, distribution, and reproduction in any medium, provided the original author(s) and source are credited.

\section{References}

[1] Hirano M, Shinjo K. Atomistic locking and friction. Phys Rev B 41(17):11837-11851 (1990)

[2] Hirano M, Shinjo K, Kaneko R, Murata Y. Anisotropy of frictional forces in muscovite. Mica Phys Rev Lett 67(19): 2642-2645(1991)

[3] Liu Z, Zhang S M, Yang J R, Liu J Z, Yang Y L, Zheng Q $\mathrm{S}$. Interlayer shear strength of single crystalline graphite. Acta Mech Sinica 28(4): 978-982 (2012)

[4] Enomoto Y, Tabor D. The frictional anisotropy of diamond. Nature 283(3):51-52 (1980)

[5] Enomoto Y, Tabor D. The frictional anisotropy of diamond. P Roy Soc Lond A Mat 373: 405-417 (1981)

[6] Hirano M, Shinjo K, Kaneko R, Murata Y. Observation of superlubricity by scanning tunneling microscopy. Phys Rev Lett 78(8):1448-1451 (1997)

[7] Martin J M, Donnet C, Lemogne T, Epicier T. Superlubricity of molybdenum-disulfide. Phys Rev B 48(14): 10583-10586 (1993)

[8] Dayo A, Alnasrallah W, Krim J. Superconductivity-dependent sliding friction. Phys Rev Lett 80(8): 1690-1693 (1998)

[9] Park J Y, Ogletree D F, Thiel P A, Salmeron M. Electronic control of friction in silicon pn junctions. Science 313(5784): 186 (2006)

[10] Erdemir A, Martin J M (Ed). Superlubricity. New York: Elsevier, 2007.

[11] Dienwiebel M, Verhoeven G S, Pradeep N, Frenken J W M, Heimberg J A, Zandbergen H W. Superlubricity of graphite. Phys Rev Lett 92(12): 126101 (2004)

[12] Dienwiebel M, Pradeep N, Verhoeven GS, Zandbergen H W, Frenken J W M. Model experiments of superlubricity of graphite. Surf Sci 576(1-3): 197-211 (2005)

[13] Feng X F, Kwon S, Park J Y, Salmeron M. Superlubric sliding of graphene nanoflakes on graphene. Acs Nano 7(2): 1718-1724 (2013)

[14] Dietzel D, Feldmann M, Schwarz U D, Fuchs H, Schirmeisen A. Scaling laws of structural lubricity. Phys Rev Lett 111(23): 235502 (2013)

[15] Deng Z, Smolyanitsky A, Li Q Y, Feng X Q, Cannara R J. Adhesion-dependent negative friction coefficient on chemically modified graphite at the nanoscale. Nat Mater 11(12): 1032-1037 (2012)

[16] Liu Z. The diversity of friction behavior between bi-layer graphenes. Nanotechnology 25(7): 075703 (2014)

[17] Urbakh M, Meyer E. Nanotribology: The renaissance of friction. Nat Mater 9(1): 8-10 (2010)

[18] Urbakh M. Friction: Towards macroscale superlubricity. Nat Nanotechnol 8(12): 893-894 (2013)

[19] Zheng Q S, Jiang B, Liu S P, Weng Y X, Lu L, Xue Q K, Zhu J, Jiang Q, Wang S, Peng L M. Self-retracting motion of graphite microflakes. Phys Rev Lett 100(6): 067205 (2008)

[20] Liu Z, Boggild P, Yang J R, Cheng Y, Grey F, Liu Y L, Wang L, Zheng Q S. A graphite nanoeraser. Nanotechnology 22(26): 265706 (2011)

[21] Liu Z, Liu J Z, Cheng Y, Li Z H, Wang L, Zheng Q S. Interlayer binding energy of graphite: A mesoscopic determination from deformation. Phys Rev B 85(20): 205418 (2012)

[22] Liu Z, Yang J R, Grey F, Liu J Z, Liu Y L, Wang Y B, Yang Y L, Cheng Y, Zheng Q S. Observation of microscale superlubricity in graphite. Phys Rev Lett 108(20): 205503 (2012)

[23] Yang J R, Liu Z, Grey F, Xu Z P, Li X D, Liu YL, Urbakh M, Cheng Y, Zheng Q S. Observation of high-speed microscale superlubricity in graphite. Phys Rev Lett 110(25): 255504 (2013)

[24] Zhang R F, Ning Z Y, Zhang Y Y, Zheng Q-S, Cheng Q, Xie H H, Zhang Q, Qian W Z, Wei F. Superlubricity in centimetres-long double-walled carbon nanotubes under ambient conditions. Nat Nanotechnol 8: 912-916 (2013)

[25] Dong Y L, Wu X W, Martini A. Atomic roughness enhanced friction on hydrogenated graphene. Nanotechnology 24(37): 375701 (2013) 
[26] Gnecco E, Maier S, Meyer E. Superlubricity of dry nanocontacts. J Phys-Condens Mat 20(35): 354004 (2008)

[27] Benedict L X, Chopra N G, Cohen M L, Zettl A, Louie S G, Crespi V H. Microscopic determination of the interlayer binding energy in graphite. Chem Phys Lett 286(5-6): 490-496 (1998)

[28] Zacharia R, Ulbricht H, Hertel T. Interlayer cohesive energy of graphite from thermal desorption of polyaromatic hydrocarbons. Phys Rev B 69(15): 155406 (2004)

[29] Park S, Floresca H C, Suh Y, Kim M J. Electron microscopy analyses of natural and highly oriented pyrolytic graphites and the mechanically exfoliated graphenes produced from them. Carbon 48(3): 797-804 (2010)

[30] Karabacak D, Kouh T, Huang C C, Ekinci K L. Optical knife-edge technique for nanomechanical displacement detection. Appl Phys Lett 88(19): 193122 (2006)

[31] Wen Q, Qian W Z, Nie J Q, Cao A Y, Ning G Q, Wang Y, Hu L, Zhang Q, Huang J Q, Wei F. 100 mm long, semiconducting triple-walled carbon nanotubes. Adv Mater 22(16): 18671871 (2010)

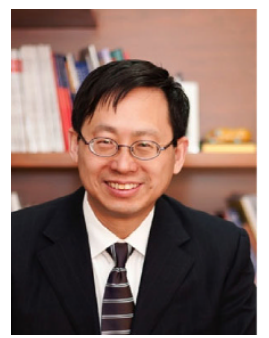

Quanshui ZHENG. He received his $\mathrm{PhD}$ degree in mechanics from Tsinghua University in 1989. Over the past ten years Zheng has been focused his research interest on interfacial motion, friction, and wet properties based on nano/micro technology and their applications for creating new classes of nano/micro devices and nano/micro fluidic tools. He and his colleagues have brought the key breakthrough for realizing superlubricity in micro and macro scales and in the ambient condition. They also pioneered the understanding on the instability and size effect of super-hydrophobicity. In 1990's, Zheng solved a few of long-lasting fundamental problems in continuum mechanics, including the establishment of the modern theory of representations for tensor functions and the invariant-based nonlinear and

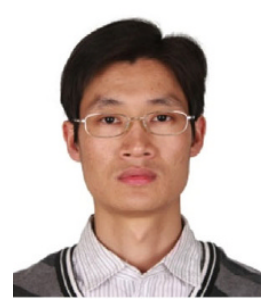

Ze LIU. He received his Bachelor degree in Mechanics from Harbin institute of Technology in 2007 and $\mathrm{PhD}$ degree in mechanics from
[32] Berman D, Erdemir A, Sumant A V. Graphene: A new emerging lubricant. Mater Today 17(1): 31-42 (2014)

[33] Erdemir A. Design criteria for superlubricity in carbon films and related microstructures. Tribol Int 37(7): 577-583 (2004)

[34] Zheng Q S, Jiang Q. Multiwalled carbon nanotubes as gigahertz oscillators. Phys Rev Lett 88(4): 045503 (2002)

[35] Zheng Q S, Liu J Z, Jiang Q. Excess van der Waals interaction energy of a multiwalled carbon nanotube with an extruded core and the induced core oscillation. Phys Rev B 65(24): 245409 (2002)

[36] Kim S Y, Cho S Y, Kim K S, Kang J W. Developing nanoscale inertial sensor based on graphite-flake with selfretracting motion. Physica E 50: 44-50 (2013)

[37] Ng T W, Lau C Y, Bernados-Chamagne E, Liu J Z, Sheridan J, Tan N. Graphite flake self-retraction response based on potential seeking. Nanoscale Res Lett 7: 1-9 (2012)

[38] Hwang H J, Kang J W. Nonvolatile graphene nanoflake shuttle memory. Physica E 56:17-23 (2014)

anisotropic constitutive equations, Eshelby problem for non-ellipsoidal inclusions, micromechanical model, and Cauchy mean rotation.

Zheng was an assistant, associate, and full professor of mechanics at the Jiangxi Polytechnic University from 1981 to 1993 . He has been a professor of mechanics at Tsinghua University since 1993, where he had serviced as the head of the engineering mechanics department (2004-2011), the founding director of the University's Center for Nano and Micro Mechanics (2010- ), and the founding director of XIN Center (2014- ), an international hub for innovation research and education co-founded by Tsinghua University and Tel Aviv University. Zheng has over five years working experience in England, France, Germany, USA, and Australia. He is also the editor-in-Chief of both Acta Mechanica Sinica and Chinese Journal of Theoretical and Applied Mechanics.

Tsinghua University in 2012. After then, he has been a postdoc in Yale Univeristy. His research interests include friction, lubrication, devices and sensors, microstructure- property relationships. 